\title{
Taxa de mortalidade de menores de 5 anos proposta pela UNICEF: análise crítica de sua validade como indicador de saúde
}

\section{Mortality rate for under-fives proposed by UNICEF: critical analysis of its vality as a health indicators}

\section{Ruy Laurenti e Jair Lício Ferreira Santos}

Departamento de Epidemiologia da Faculdade de Saúde Pública da Universidade de São Paulo. São Paulo, SP - Brasil

\begin{abstract}
Resumo
Investiga-se o relacionamento entre a taxa de mortalidade de menores de cinco anos (TMM5) e os indicadores clássicos - mortalidade infantil, mortalidade de menores de 1 ano, de 1 a 4 anos, menores de cinco anos e probabilidade de óbito entre 0 e 5 anos $\left({ }_{5} q_{0}\right)$. Do ponto de vista teórico é mostrada a equivalência entre a TMM5 e a probabilidade ${ }_{5} \mathrm{q}_{0}$ da tábua de vida. Para a avaliação prática da TMM5 utilizam-se indicadores obtidos para as 24 áreas das Américas que participaram da Investigação Inter-americana de Mortalidade na Infância. A equivalência teórica entre a taxa (TMM5) e ${ }_{5} q_{0}$ é mostrada por meio do diagrama de Lexis. As comparações feitas através do Coeficiente de Correlação de Kendall mostram a alta concordância, principalmente entre a TMM5 e o Coeficiente de Mortalidade de menores de cinco anos e o Coeficiente de Mortalidade Infantil.
\end{abstract}

Coeficiente de mortalidade. Mortalidade infantil.

\begin{abstract}
The relationship between the Mortality Rate for children under age 5 as proposed by UNICEF and classical mortality indicators - infant mortality, mortality rates for children under age 1, for ages 1 to 4 , mortality for children under 5, and the probability of death from birth to 5 years of age, is evaluated. The theoretical equivalence between the rate and ${ }_{5} q_{0}$ is shown by means of a Lexis diagram. Pratical evaluation is carried out using data from the 24 areas under analysis in the Inter-American Investigation of Mortality in Childhood. Kendall's Correlation shows high agreement between the rate and classical indicators, mainly for the Coefficient for children under 5 and for infant mortality.
\end{abstract}

Mortality rate. Infant mortality. 


\section{INTRODUÇÃO}

Os indicadores de saúde, particularmente aqueles baseados em dados de mortalidade, vêm, há muito tempo, sendo utilizados para diagnosticar situações e apontar prioridades. Dentre eles destacam-se a taxa de mortalidade infantil e as taxas de mortalidade de menores de 1 ano e de 1 a 4 anos. Esses indicadores são muito úteis principalmente em áreas pouco desenvolvidas como na maioria dos países latino-americanos, africanos e asiáticos. Ocorre que a construção desses indicadores, na maioria dessas regiões, freqüentemente está bastante prejudicada devido a má qualidade dos registros de nascidos vivos e mesmo de óbitos, principalmente para menores de 1 ano. Assim, não raro, baixas taxas de mortalidade infantil podem ser resultantes de sub-registro de óbito e não o reflexo de boas condições de saúde da população infantil. Por outro lado, altas taxas podem estar refletindo muito mais um sub-registro de nascimento do que, na realidade, más condições de saúde.

A necessidade de se ter um indicador que não estivesse sujeito aos problemas mencionados levou a UNICEF a solicitar à Divisão de População das Nações Unidas a criação de um indicador que retratasse a morte de crianças e não estivesse sujeito a sub-registros. Foi então apresentado um novo índice, que passou a ser conhecido como TMM5 ou a taxa de mortalidade de menores de 5 anos.

\section{TMM5 - Taxa de Mortalidade de Menores de 5 anos}

A publicação da UNICEF “Situação Mundial da Infância, 1987" 3 dá destaque a um "Novo Índice de Mortalidade" descrevendo o que se segue: "Como resultado da colaboração entre a Divisão de População das Nações Unidas e a UNICEF é possível, agora, apresentar um novo índice, mais aprimorado, de mortalidade infantil para menores de um ano e préescolares - a taxa de mortalidade de menores de 5 anos (TMM5)".

Continuando, a citada publicação da UNICEF afirma:

"A mortalidade de crianças, nas publicações internacionais, tem sido até aqui explicada estatisticamente, como mortes por 1.000 crianças com idade variando entre 1 e 4 anos, inclusive. Esse dado usa um denominador diferente daquele utilizado para as taxas de mortalidade de menores de 1 ano (mortes por 1.000 nascidos vivos). Isto significa que as duas estimativas não poderiam ser facilmente combinadas e analisadas. Além disso, como o denominador usado para a taxa de mortalidade pré-escolar é muito alto, a taxa rapidamente cai para menos de um, à medida em que o país melhora suas condições de saúde... Espera-se que a TMM5 (taxa de mortalidade de menores de 5 anos) venha a ser adotada pelos países, nas análises nacionais e regionais e para a apresentação nos próximos anos, de modo que possa rapidamente se tornar a forma padrão quando se discutir mortalidade infantil".

$\mathrm{A} \mathrm{UNICEF}^{3}$ dá a seguinte definição para TMM5: é a relação entre o número de óbitos de menores de 5 anos num determinado ano e o número de nascidos vivos naquele ano.

Chama a atenção o fato de que, como está expressa, não é uma "taxa", seria mais uma "razão". Por outro lado qual o significado estatístico desta razão?

A publicação das Nações Unidas "Mortality of Children under age $5 "{ }^{4}$ descreve a metodologia do cálculo da TMM5 e a aplicação para calcular as estimativas e projeções mundiais para o período 19502025.

A finalidade do projeto levado a efeito pelo "Department of International Economic and Social Affairs", das Nações Unidas, foi produzir série de estimativas de mortalidade infantil e de menores de 5 anos, medidas como a probabilidade de morrer antes da idade 1 , a probabilidade de morrer entre as idades 1 e 5 e a probabilidade de morrer antes da idade 5 . Isso para períodos passados e, baseando-se nessas séries, projetar os futuros níveis para cada país. As estimativas e projeções destas probabilidades aparecem para períodos qüinqüenais entre 1950 e 2025.

Para os países onde o registro civil tem boa cobertura é fácil calcular a mortalidade infantil, a mortalidade de 1 a 4 anos e a mortalidade de menores de 5 anos. Para esses mesmos países a probabilidade de morrer entre as idades 1 e $5\left({ }_{4} q_{1}\right)$ e antes da idade $5\left({ }_{5} \mathrm{q}_{0}\right)$ são calculados pela metodologia da tábua de vida.

Sabe-se, porém, que para os países onde a cobertura do registro civil é muito baixa, e pelo que ocorre em quase todos os países subdesenvolvidos, não é possível calcular as taxas de mortalidade e, da mesma maneira, é impossível calcular as probabilidades de morrer por meio do método da tábua de vida. Para ambos os tipos de medidas é preciso recorrer aos dados coletados nos censos e diferentes tipos de inquéritos ("surveys").

A descrição da metodologia nesses casos, para países subdesenvolvidos, está apresentada na citada publicação das Nações Unidas ${ }^{4}$ e em parte é derivado do chamado método de Brass ${ }^{2}$. 
O que chama a atenção de muitos que têm trabalhado com indicadores de saúde é o fato de que a UNICEF apresenta uma TMM5 definida como o número de óbitos de menores de 5 anos em um determinado ano $\mathrm{x}$ e o número de nascidos vivos no mesmo ano $\mathrm{x}$. O que pode parecer, em primeira análise, ser diferente da probabilidade de morrer até completar 5 anos $\left({ }_{5} \mathrm{q}_{0}\right)$ como apresenta as Nações Unidas.

Por outro lado, vem preocupando muitos profissionais de saúde o fato de que se está disseminando o uso da TMM5, particularmente em áreas pobres, onde se sabe ser grande o sub-registro de nascimento. Nesses casos, dever-se-ia obter a TMM5 pelo ${ }_{5} \mathrm{q}_{0}$ como propõe o método de Brass.

O presente trabalho propõe-se a analisar três seguintes aspectos em relação a TMM5:

1. Explicitar o relacionamento técnico existente entre a TMM5 e a probabilidade de morrer entre 0 e 5 anos de idade, ${ }_{5} q_{0}$, bem como as eventuais aproximações envolvidas.

2. Verificar em situações em que se dispõe de bons registros de nascimentos e óbitos a TMM5, calculada como propõe a UNICEF, se o comportamento dos indicadores clássicos (mortalidade infantil, mortalidade de 1 ano de idade, de 1 a 4 anos e de menores de 5 anos) é o mesmo.

3. Da mesma forma, verificar se nas situações em que se dispõe de bons registros de nascimentos e óbitos, a TMM5 calculada, como propõe a UNICEF, representa ou não uma boa aproximação para a probabilidade de morrer entre o nascimento e os cinco anos de idade, ${ }_{5} \mathrm{q}_{0}$, vigente na população.

\section{METODOLOGIA}

Para os objetivos 2 e 3 tomaram-se os dados de nascimentos vivos, óbitos de menores de 1 ano, 1 ano, 1 a 4 anos e menores de 5 anos para 24 áreas das Américas que participaram do projeto "Inter-american Investigation of Mortality in Childhood" ${ }^{1}$. Entre essas áreas havia aquelas com baixas taxas de mortalidade infantil e de crianças de 1 a 4 anos de idade, como São Francisco, (Califórnia) e Sherbrooke (Canadá) e outras com alta mortalidade, como Viacha (Bolívia), Recife (Brasil), Departamentos Rurais da Povíncia do Chaco na Argentina. Entre os dois extremos havia áreas intermediárias.

Dentre essas 24 áreas havia algumas com bons registros de nascimento e óbitos e outras com registros deficientes, sendo que em algumas delas o sub-registro era muito grande. Ocorre, porém, que pela própria metodologia da investigação foram coletados, de várias maneiras, os dados sobre nascidos vivos e óbitos durante o período de estudo que foi de 24 meses. Portanto, é possível afirmar, com quase certeza, que foram coletados todos os dados necessários para o cálculo das taxas de tal maneira que as 24 áreas se comportaram para as finalidades aqui desejadas, como áreas de bom registro e nas quais se conheciam muito bem os dados vitais.

Para as 24 áreas foram calculadas as taxas da seguinte maneira:

MI = Mortalidade Infantil =

$$
=\frac{\text { óbitos menores de } 1 \text { ano }}{\text { número de nascidos vivos }} \times 1.000
$$

$\mathrm{M}_{\mathrm{O}}=$ Mortalidade de 1 ano de idade =

$$
=\frac{\text { óbitos de } 1 \text { ano de idade }}{\text { população de } 1 \text { ano de idade }} \times 1.000
$$

${ }_{4} \mathrm{M}_{1}=$ Mortalidade de 1 a 4 anos de idade $=$

$$
=\frac{\text { óbitos de } 1 \mathrm{a} 4 \text { anos de idade }}{\text { população de } 1 \text { a } 4 \text { anos de idade }} \times 1.000
$$

${ }_{5} \mathrm{M}_{0}=$ Mortalidade de menores de 5 anos de idade $=$ $=\frac{\text { óbitos de menores de } 5 \text { anos de idade }}{\text { populção de menores de } 5 \text { anos de idade }} \times 1.000$

TMM5 $=\frac{\text { óbitos de menores de } 5 \text { anos }}{\text { número de nascidos vivos no }} \times 1.000$

${ }_{5} \mathrm{q}_{0}=$ Probabilidade de morrer entre 0 e 5 anos $=$

$$
\begin{aligned}
& q_{0}+{ }_{4} q_{1}-q_{04} q_{1} \mathrm{e} \\
& q_{0}=\frac{M_{0}}{1+0,8 \cdot M_{0}}, \quad{ }_{4} q_{1}=\frac{4 \cdot{ }_{4} M_{1}}{1+2,8_{4} M_{1}}
\end{aligned}
$$

Para relacionar a TMM5 como previsto no segundo objetivo, será utilizado um diagrama de Lexis.O grau de concordância da TMM5 com as demais medidas, como previsto nos dois outros objetivos, será avaliado pelo coeficiente de correlação por postos de Kendall ${ }^{2}$.

\section{RESULTADOS}

Pelo diagrama de Lexis (Figura) vê-se que os óbitos de menores de 5 anos ocorridos no ano a (isto é, dentro do retângulo de linhas contínuas) são compostos de duas proporções $\alpha$ e $\beta$, tais que $\alpha+\beta=1$.

Uma parte " $\alpha$ " dos óbitos que nasceram no ano $\underline{\mathrm{a}-\mathrm{X}}$ completou $\mathrm{x}$ anos em $\underline{\mathrm{a}} \mathrm{e}$ morreram naquele ano. Outra parte " $B$ " referente aos que nasceram no ano $\underline{\mathrm{a}-\mathrm{x}}$, completaram $\mathrm{x}$ anos em $\underline{\mathrm{a}-1}$ e morrem com essa idade em $\underline{a}$.

Igualmente, os que nasceram no ano a-1 morrem numa proporção $\alpha$ no mesmo ano e $\beta$ no ano a. $\mathrm{O}$ mesmo vale para os que nasceram em a.

Seja $\mathrm{p}(\mathrm{x})$ a verdadeira probabilidade de sobreviver do nascimento à idade $\mathrm{x}$. O número de óbitos em a, de crianças dessa idade será:

$$
\begin{aligned}
& D_{(x)}=N(a-x) q_{x} \alpha+N(a-x-1) p(x) q_{x} \beta \\
& \text { onde } \mathrm{N}(\mathrm{a}-\mathrm{x})=\text { nascidos vivos em } \mathrm{a}-\mathrm{x} \\
& q_{x}=\text { verdadeira probabilidade de óbitos entre } x \text { e }
\end{aligned}
$$




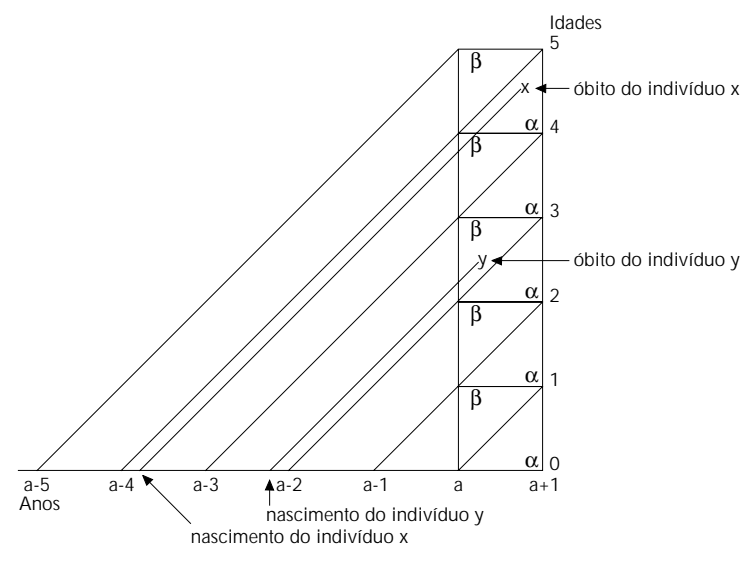

Figura - Diagrama de Lexis relacionando os óbitos ocorridos no ano 'a' com os nascimentos do mesmo ano e de anos anteriores.

Se as condições de mortalidade não sofrerem bruscas alterações de nível de um ano para outro (condição 1$)$, os valores de $\mathrm{p}(\mathrm{x})$ nos dois termos de $\mathrm{D}(\mathrm{x})$ serão os mesmos.

Por outro lado, se o número de nascimentos for razoavelmente estável (condição 2), ter-se-á:

$$
\mathrm{N}(\mathrm{a})=\mathrm{N}(\mathrm{a}-1)=\mathrm{N}(\mathrm{a}-2) \ldots=\mathrm{N}(\mathrm{a}-\mathrm{x}),
$$

e poderemos escrever, denominando ${ }_{5} 0_{0} \mathrm{o}$ número de óbitos na população de 0 a 5 anos no ano a:

${ }_{5} 0_{\mathrm{o}}=\sum_{x=0}^{5} N(a) p(x)\left\{q_{x} \alpha+q_{x} \beta\right\}=N(\alpha) \sum_{x=0}^{5} p(x) q x$,

já que $\alpha+\beta=1$

Mas p(x).qx nada mais é do que $\frac{l(x)}{l \cdot} \cdot q x$ e $l(x) \cdot q x=5^{d} x$, o número de óbitos de 0 a 5 anos numa tábua de vida que refletisse as condições de mortalidade da população real. Ter-se-á, portanto, para a TMM5

$$
\text { TMM 5 }=\frac{5^{0} o}{N(a)}=\frac{N(a) \sum_{x=0}^{5} \frac{5}{l o} \frac{5^{d}}{l o}}{N(a)}=\frac{\sum 5 d x}{l o}=5^{q} 0
$$

Isto é, a TMM5 é numericamente igual à probabilidade de morrer entre 0 e 5 anos de idade, sob as condições 1 e 2 antes mencionadas.

A Tabela 1 mostra que a TMM5 tem comportamento semelhante às taxas de mortalidade infantil e de crianças menores de 5 anos.

Os coeficientes de correlação calculados para as comparações da TMM5 com as outras quatro taxas mostraram os valores expressos na Tabela 2.

Verificam-se altos valores de concordância entre TMM5 e as outras medidas, todos significantes, sen- do o maior deles entre a TMM5 e o coeficiente de mortalidade para menores de 5 anos.

O coeficiente de correlação não é tão alto para ${ }_{5} \mathrm{q}_{0}$, decorrente do fato de que esta resulta da combinação de q0 e ${ }_{4} q_{1}$, também com coeficientes da ordem de 0,76 .

Pelos dados empíricos, conclui-se que a TMM5 reproduz com forte concordância as ordenações de nível de mortalidade dados pela mortalidade infantil e, sobretudo, pelo coeficiente para menores de 5 anos.

\section{COMENTÁRIOS}

Quando se dispõe de boa cobertura das mortes infantis e de crianças até 5 anos de idade, bem como de bom registro de nascimento, a TMM5 calculada como propõe a UNICEF tem comportamento semelhante aos indicadores clássicos que medem as condições de saúde daqueles grupos etários. Calcular a TMM5 para áreas pobres, com péssimos registros vitais, baseando-se nesses dados, é um erro grave. Ela não deve ser calculada pois estará sujeita aos mesmos erros que o cálculo da mortalidade infantil e mortalidade de crianças menores de 5 anos.

Por outro lado, quando há bons registros assim como bons dados de população qual a vantagem de calcular a TMM5? Qual a vantagem sobre os outros indicadores?

Demonstrou-se que a TMM5 representa uma boa aproximação para a probabilidade de morrer entre o nascimento e os cinco anos de idade vigente na população. Essa seria uma vantagem de se calcular a TMM5 quando há boa cobertura de registro vital.

Quando não se dispõe de bons dados, o cálculo da TMM5 deve ser feito pelo método de Brass. Isso teria uma vantagem adicional: naquelas populações onde devido a precariedade dos registros as informações da mortalidade são obtidas pela técnica de Brass, pode-se fazer uma comparação imediata da TMM5 com outras populações por meio da sobrevivência dos filhos de mulheres de 30 a 35 anos. É preciso, porém, lembrar que ${ }_{50}^{\mathrm{q}}$ obtido pela técnica de Brass representa uma média de condições da mortalidade, cujo centro está cerca de 3 anos antes da data da pesquisa.

Concluindo, pode-se afirmar que a TMM5, calculada como recomenda a UNICEF, para áreas com bom registro, tem o mesmo valor que os indicadores clássicos de mortalidade de crianças. É 
Tabela 1 - Valores de TMM 5 e medidas de mortalidade para grupos de menores de 5 anos em áreas da investigação de mortalidade na infância.

\begin{tabular}{|c|c|c|c|c|c|c|}
\hline \multirow{4}{*}{ Local* } & \multicolumn{6}{|c|}{ M edidas de M ortalidade } \\
\hline & \multirow[t]{2}{*}{ TM M 5} & & \multicolumn{2}{|c|}{ Coeficiente de Mortalidade } & \multicolumn{2}{|c|}{ Probabilidadee } \\
\hline & & Infantil & 1 Ano & 1-4 Anos & $>5$ Anos & \\
\hline & $1.000 \mathrm{nv}$ & $1.000 \mathrm{nv}$ & $1.000 \mathrm{H}$ & $1.000 \mathrm{Hab}$. & $1.000 \mathrm{Hab}$. & ${ }_{5} q_{0} 1.000$ exp. \\
\hline 1 & 19,9 & 17,2 & 0,7 & 0,7 & 4,1 & 3,5 \\
\hline 2 & 20,7 & 18,5 & 0,8 & 0,8 & 5,4 & 4,0 \\
\hline 3 & 21,8 & 18,3 & 1,2 & 0,8 & 4,1 & 4,4 \\
\hline 4 & 46,4 & 38,8 & 4,4 & 2,2 & 10,5 & 13,1 \\
\hline 5 & 49,8 & 43,0 & 3,9 & 1,9 & 10,9 & 11,4 \\
\hline 6 & 56,0 & 50,7 & 4,0 & 1,5 & 12,9 & 9,9 \\
\hline 7 & 59,4 & 50,8 & 3,6 & 2,4 & 13,0 & 13,1 \\
\hline 8 & 61,9 & 54,9 & 4,0 & 1,8 & 13,0 & 11,1 \\
\hline 9 & 66,2 & 57,9 & 5,4 & 2,2 & 14,0 & 14,1 \\
\hline 10 & 69,5 & 47,6 & 12,5 & 5,7 & 14,4 & 34,5 \\
\hline 11 & 70,1 & 47,8 & 15,5 & 5,8 & 14,6 & 37,8 \\
\hline 12 & 74,1 & 65,1 & 5,2 & 2,8 & 17,7 & 16,2 \\
\hline 13 & 74,6 & 60,7 & 9,4 & 5,3 & 18,1 & 30,0 \\
\hline 14 & 77,0 & 54,6 & 13,4 & 5,9 & 16,1 & 36,2 \\
\hline 15 & 85,3 & 71,5 & 6,9 & 4,0 & 19,4 & 22,6 \\
\hline 16 & 88,2 & 76,2 & 9,3 & 3,6 & 20,7 & 23,4 \\
\hline 17 & 98,5 & 87,9 & 7,0 & 2,9 & 21,9 & 18,4 \\
\hline 18 & 106,8 & 81,7 & 17,4 & 8,0 & 26,4 & 47,9 \\
\hline 19 & 107,3 & 85,0 & 16,3 & 6,3 & 23,9 & 40,5 \\
\hline 20 & 109,1 & 94,5 & 9,4 & 4,0 & 24,0 & 25,0 \\
\hline 21 & 111,9 & 73,0 & 27,2 & 11,9 & 26,6 & 71,5 \\
\hline 22 & 119,6 & 91,2 & 19,2 & 9,0 & 29,3 & 53,4 \\
\hline 23 & 189,4 & 123,5 & 50,7 & 21,7 & 48,1 & 126,6 \\
\hline 24 & 200,4 & 120,0 & 48,1 & 26,2 & 50,5 & 139,4 \\
\hline
\end{tabular}

Fonte: Puffer e Serrano ${ }^{1}$ (1973).

* - 1. Regiões suburbanas de São Francisco, USA; 2. São Francisco, USA; 3. Sherbrooke, Canadá; 4. Kingston/St. Andrew, Jamaixa; 5 . Ribeirão Preto, Brasil; 6. S. Juan (urbano), Argentina; 7. Comunidades de Batatais, Brodosqui, Cravinhos, Jardinópolis e Sertãozinho, Brasil; 8. Santiago, Chile; 9. Áreas suburbanas e rurais de Santiago, Chile; 10. M edelin, Colombia; 11. Cartagena, Colombia; 12. São Paulo, Brasil; 13. M onterrey, México; 14. Cali, Colombia; 15. Franca, Brasil; 16. Resistência (urbano), Argentina; 17. Departamento suburbano Província San Juan, Argentina, 18. San Salvador (urbano), El Salvador; 19. Departamentos rurais, Província El Chaco, Argentina; 20. Departamentos rurais, Província San Juan, Argentina; 21. La Paz, Bolívia; 22. Recife, Brasil; 23. Viacha, Bolívia; 24. Municípios rurais de El Salvador, El Salvador.

Tabela 2 - Coeficientes de Correlação de Kendall, entre TM M 5 e outras medidas e valores observados de $Z$ para 0 teste de significância.

\begin{tabular}{lcc}
\hline Medidas & Coeficiente & Z \\
\hline Mortalidade infantil & 0,83 & 5,71 \\
Coeficiente para menores de 1 ano & 0,76 & 5,23 \\
Coeficiente para idades 1 a 4 & 0,76 & 5,19 \\
Coeficiente para menores de 5 anos & 0,97 & 6,66 \\
Probabilidade de morrer entre 0 e & & \\
5 anos & 0,76 & 5,22 \\
\hline
\end{tabular}

Valor crítico para prova monocaudal, 5\%; 164 . importante saber que ela representa uma boa aproximação com a probabilidade de morrer entre o nascimento e os 5 anos de idade vigente na população.

Em áreas onde a cobertura do registro de nascimentos vivos e de mortes não é boa não se deve calcular a TMM5 da maneira simples como recomenda a UNICEF. Nesses casos, por meio de "surveys" ou censos calcula-se a TMM5, no caso o ${ }_{5} \mathrm{q}_{0}$, obtida, pelo método de Brass.

\section{REFERÊNCIAS BIBLIOGRÁFICAS}

1. PUFFER, R.R. \& SERRANO, C.V. Patterns of mortality in childhood. Washington, D.C., 1973. (PAHO - Scientific Publication - 262).

2. SIEGEL, S. Estatística não paramétrica. São. Paulo, Mc Graw Hill, 1975
3. UNICEF. Division of Information and Public Affairs. Situação mundial da infância, 1987. New York, UNICEF House, 1987.

4. UNITED NATIONS. Department International Economic and Social Affairs. Mortality of children under age 5: world estimates and projetions, 1950-2025. New York, 1988. (Population Studies 105). 